\title{
ИЗОЛЯЦИЯ УСТЬЕВ ЛЕГОЧНЫХ ВЕН ОДНОМОМЕНТНО С АБЛАЦИЕЙ КАВАТРИКУСПИДАЛЬНОГО ПЕРЕШЕЙКА БЕЗ ИСПОЛЬЗОВАНИЯ РЕНТГЕНОСКОПИИ
}

\begin{abstract}
PULMONARY VEIN ISOLATION
SIMULTANEOUS WITH CAVATRICUSPID
ISTHMUS ABLATHION: ZERO FLUORO
APPROACH
N. Safonov

V. Baranovich

A. Faibushevich

Summary. The article considers the problem of carrying out electrophysiological procedures without using X-ray. The work uses the results of PVI at the same time with ablation of CTI with system three-dimensional navigation, without using fluoroscopy. The study was conducted in 2019. The study included 32 patients. Average age $63 \pm 3$ years. The procedures were carried out using a navigation system. Puncture of the interatrial septum and control of the position of the instruments was carried out using intracardiac ultrasound. The first step of the procedure was pulmonary vein isolation, followed by ablation of the CTI. Follow-up period was 12 months. No cases of typical atrial flutter were diagnosed. 6 patients had AF episodes 4 months after surgery. Conclusion: Conducting a combined operation, namely ablation of CTI with pulmonary vein isolation, without the use of fluoroscopy, is possible and safe.
\end{abstract}

Keywords: atrial fibrillation, typical atrial flutter, radiofrequency ablation, fluoroscopy.

\section{Введение}

B настоящее время в интервенционном лечении нарушений ритма сердца широкие применение нашли флюороскопические системы трехмерного электроанатомического картирования для облегчения процедур катетерной абляции. Данной проблеме посвящено множество исследований $[1,2,3,4,5,6$, $7,8,10]$. Однако в данные работы не были включены
Сафонов Никита Владимирович

Aспирант, ФГАОУ ВО «Российский университет

дружбы народов» safon_92@mail.ru

Владислав Юрьевич Баранович

К.м.н., ФГАОУ ВО «Российский университет дружбы

народов»

vubara@yandex.ru

Файбушевич Александр Георгиевич

К.м.н., ФГАОУ ВО «Российский университет дружбы народов»

faybushevich_ag@pfur.ru

Аннотация. Статья затрагивает проблему проведения электрофизиолоигческих процедур без использования рентгеносокпии. В работе представлены результаты проведения изоляции устьев легочных вен одномоментно с аблацией КТП с использованием систем трехмерной навигации, без использования рентогеноскопии. Исследование проводилось в 2019 году. В исследование включено 32 пациента. Средний возраст $63 \pm 3$ года. Процедуры выполнялись с помощью навигационной системы. Пункция межпредсердной перегородки и контроль положения инструментов осуществлялся с помощью внутрисердечного УзИ. Первым этапом выполнялась изоляции легочных вен, далее абляция КТИ. Период отдаленного наблюдения составил 12 месяцев. Случаев типичного трепетания предсердий диагностировано не было. У 6 пациентов были зарегистрированы эпизоды ФП спустя 4 месяца после операции. Заключение: Проведение сочетанной операции, а именно абляции КТП с изоляцией легочных вен без использования рентгеноскопии возможно и безопасно.

Ключевые слова: фибрилляции предсердий, типичное трепетание предсердий, радиочастотная абляция, рентгеноскопия.

пациенты с ТП. Также имеются работы о проведении процедур катероной аблации без использования нефлюороскопических методов при лечении суправентриклярных тахикардий за исключением фибрилляции предсердий и атипичного трепетания предсердий $[9$, 11]. Большинство интервенционных аритмологов проводят абляцию каватрикуспидального перешейка (КТП) с использованием рентгеноскопии. В нашей работе мы предоставим результаты проведения изоляции легоч- 
Таблица 1. Сопутствующие заболеваниям и факторы риска возникновения типичного трепетания предсердий»

\begin{tabular}{|l|l|}
\hline Количество пациентов / patients & $\mathbf{3 2 1 0 0 \%}$ \\
\hline Мужчины / male & $1650 \%$ \\
\hline Женщины / female & $1650 \%$ \\
\hline Сахарный диабет /diabetes mellitus & $618,5 \%$ \\
\hline Хроническая сердечная недостаточность / chronic heart failure & $39,38 \%$ \\
\hline Удлинённый интервал р-q / increased p-q interval & $412,5 \%$ \\
\hline Ишемическая болезнь сердца / ischemic heart disease & $515,63 \%$ \\
\hline Хроническая обструктивная болезнь легких / chronic obstructive pulmonary disease & $26,25 \%$ \\
\hline Артериальная гипертония / arterial hypertension & $1856,25 \%$ \\
\hline Курение / sтокіng & $618,75 \%$ \\
\hline Хроническая болезнь почек / chronic kidney disease & $39,38 \%$ \\
\hline Ожирение / оbеsity & $412,5 \%$ \\
\hline
\end{tabular}

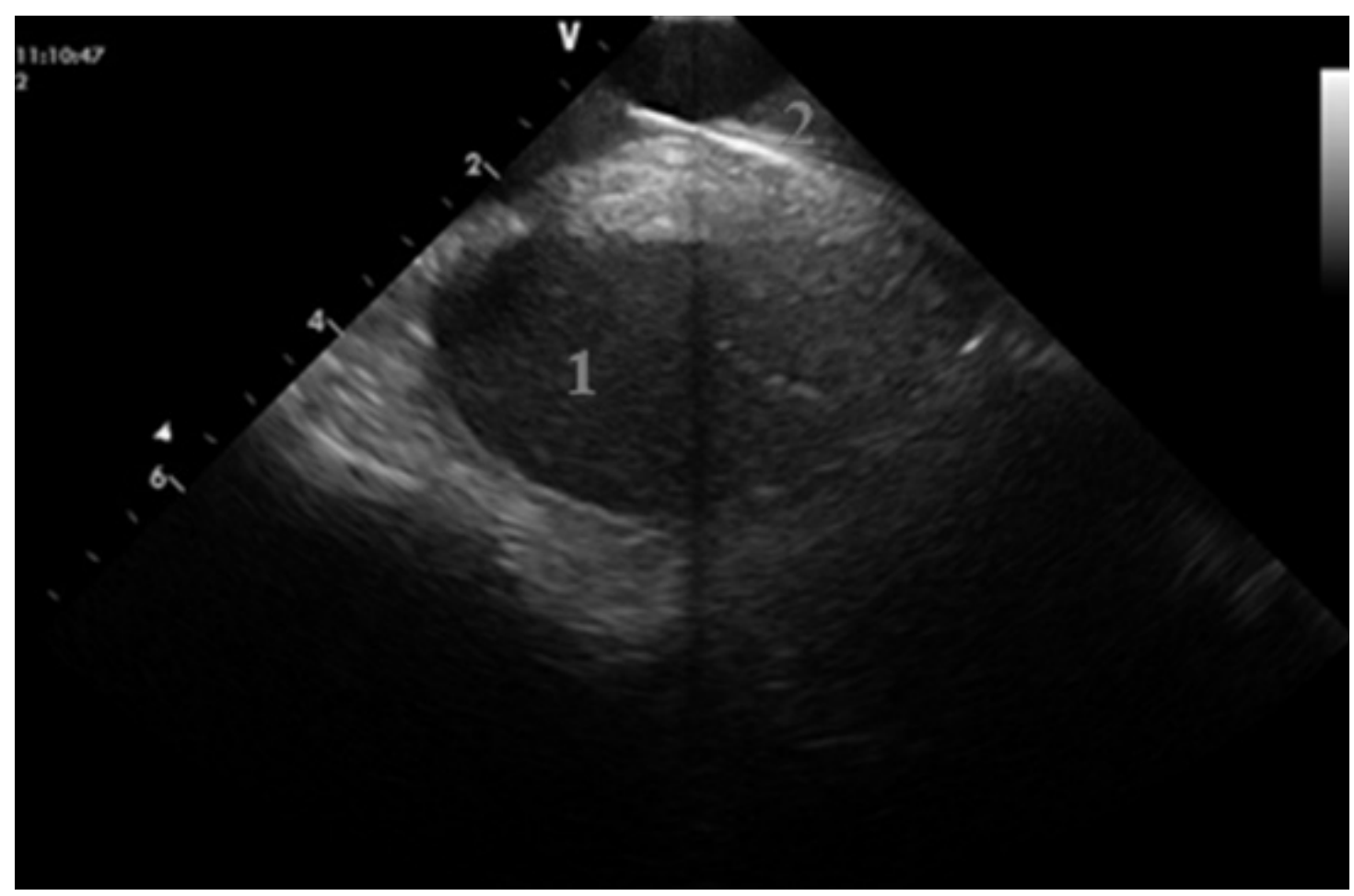

Рис. 1. Позиционирование проводников в области ВПВ

Примечание: 1 - полость ЛП, 2 проводники в ВПВ. Note: 1 - left atrium, 2 - guidewires in vena cava superior.

ных вен одномоментно с абляцией КТП, с помощью внутрисердечной эхокардиографии, без использования рентгеноскопии.

\section{Le^ь}

Оценить результаты проведения изоляции легочных вен одномоментно с абляцией КТП без использования рентгеноскопии.

\section{Материалы и метоны}

Наша работа представляет собой пилотный проект. Работа проводилось в 2019 году. Критерии включения: пароксизмальная ФП, наличие документированного ТП. Критерии исключения: персистирующая ФП, отсутствие ТП в анамнезе. Конечные точки: основные сердечно-сосудистые события, ре-операции, регистрация пароксизмов ТП. В работу включено 32 пациента. 


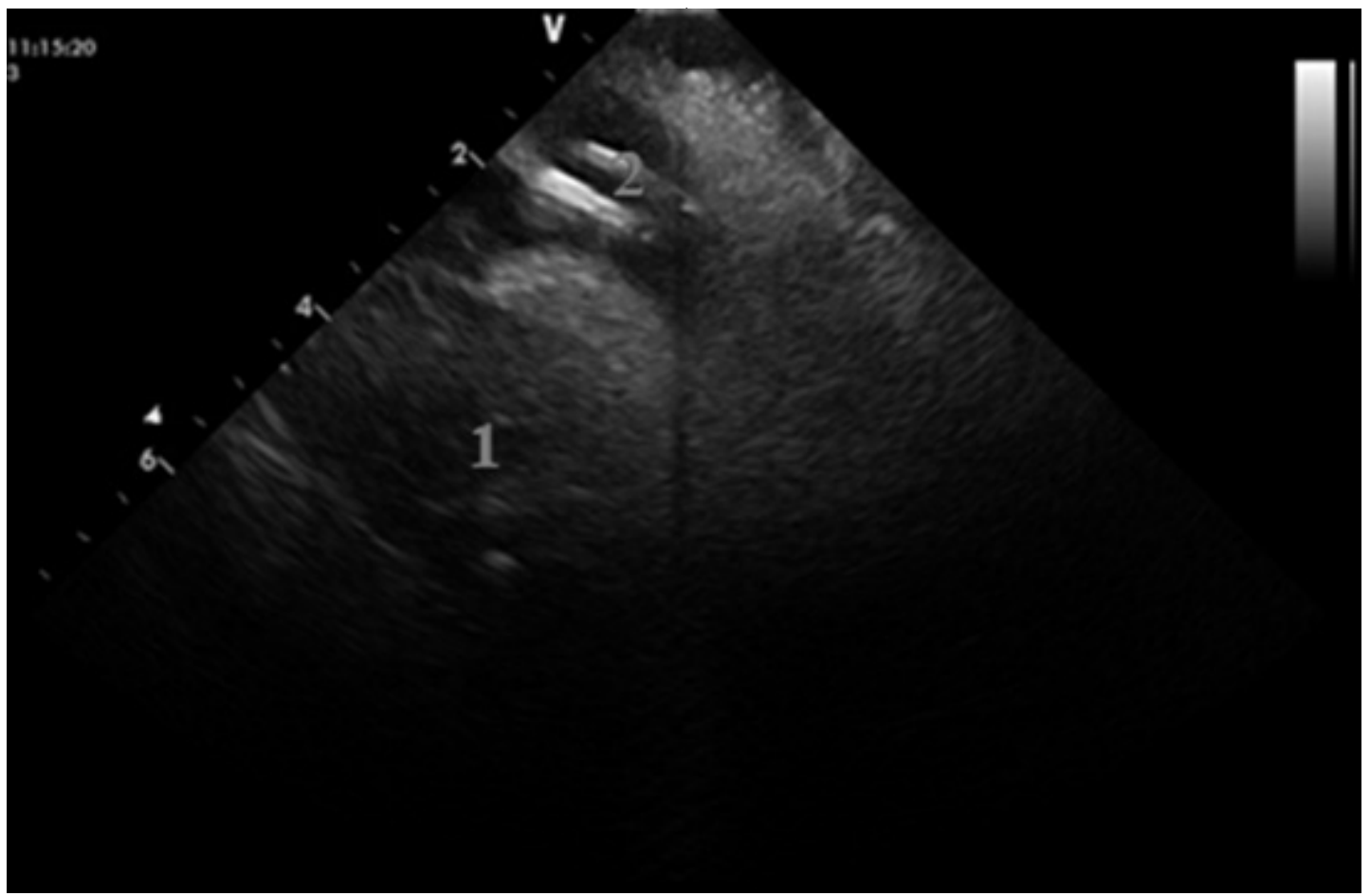

Рис. .2 Позиционирование интродьюсеров в ВПВ

Примечание: 1 - полость ЛП, 2 интродьюсеры в ВПВ. Note: 1 - left atrium, 2-2 introducer in vena cava superior.

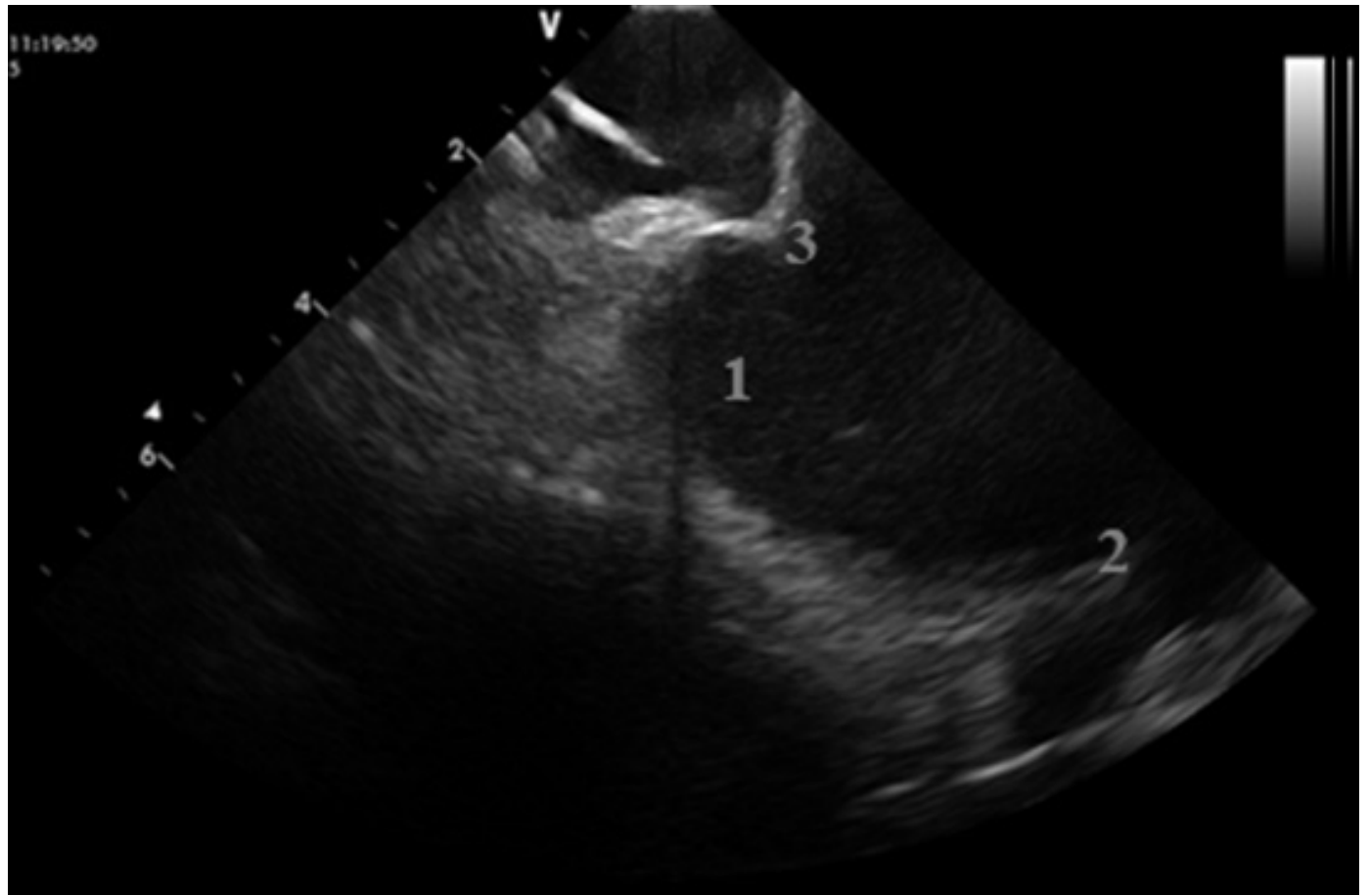

Рис. 3. Место пункции МПП

Примечание 1 - полость ЛП, 2 карина между левыми легочными венами, 3 положение позиция транссептальной иглы, натяжение МПП. Note: 1 - left atrium, 2 - carina between left pulmonary veins, 3 - tenting of interatrial septum 


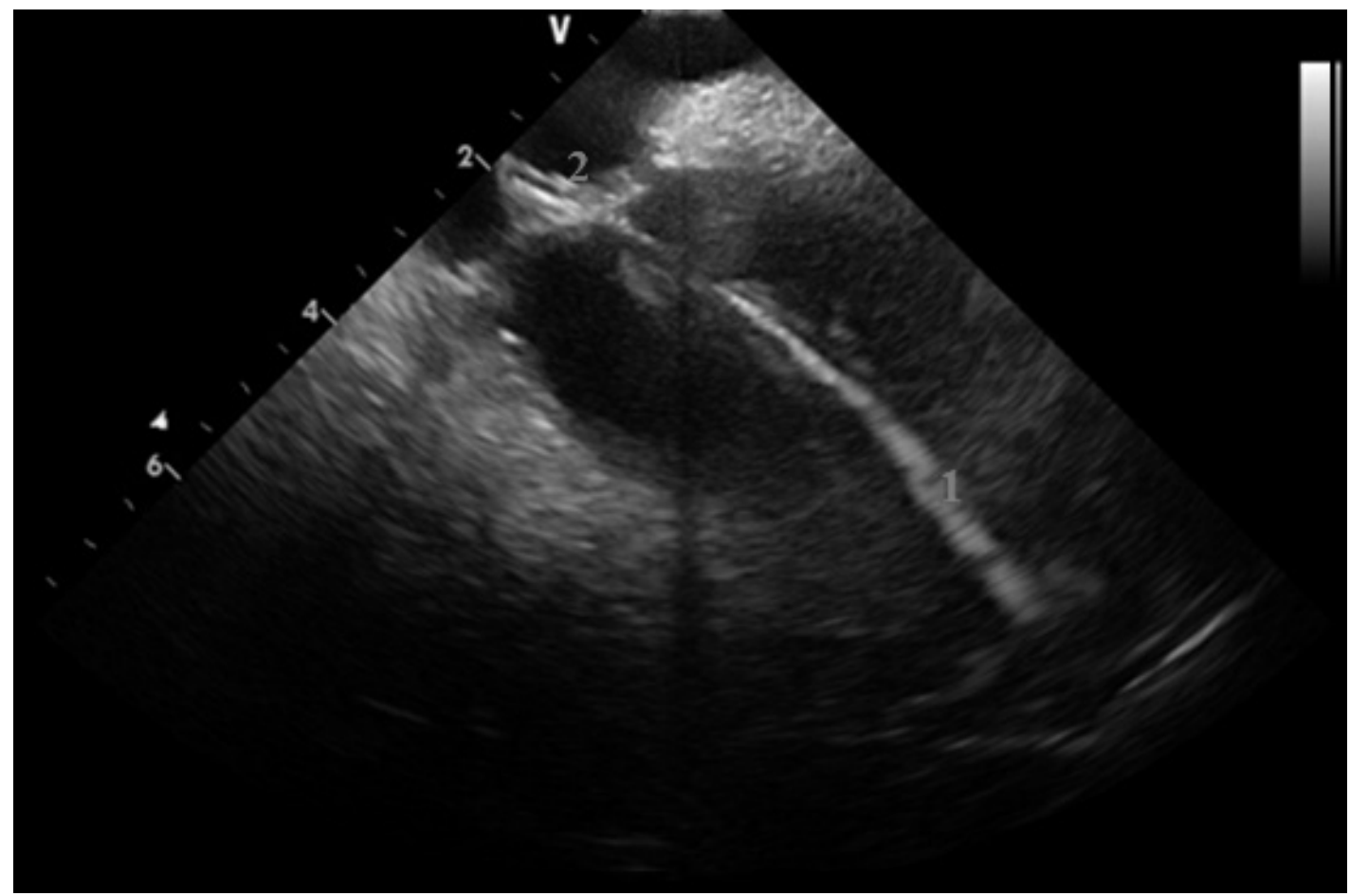

Рис. 4. Проводник в области левой верхней легочной вены

Примечание 1 - проводник в левой верхней легочной вене, 2 - комплекс дилятор в интрольюсере. Note: 1 - guidewire in left superior pulmonary vein, 2 - dilator and introducer.

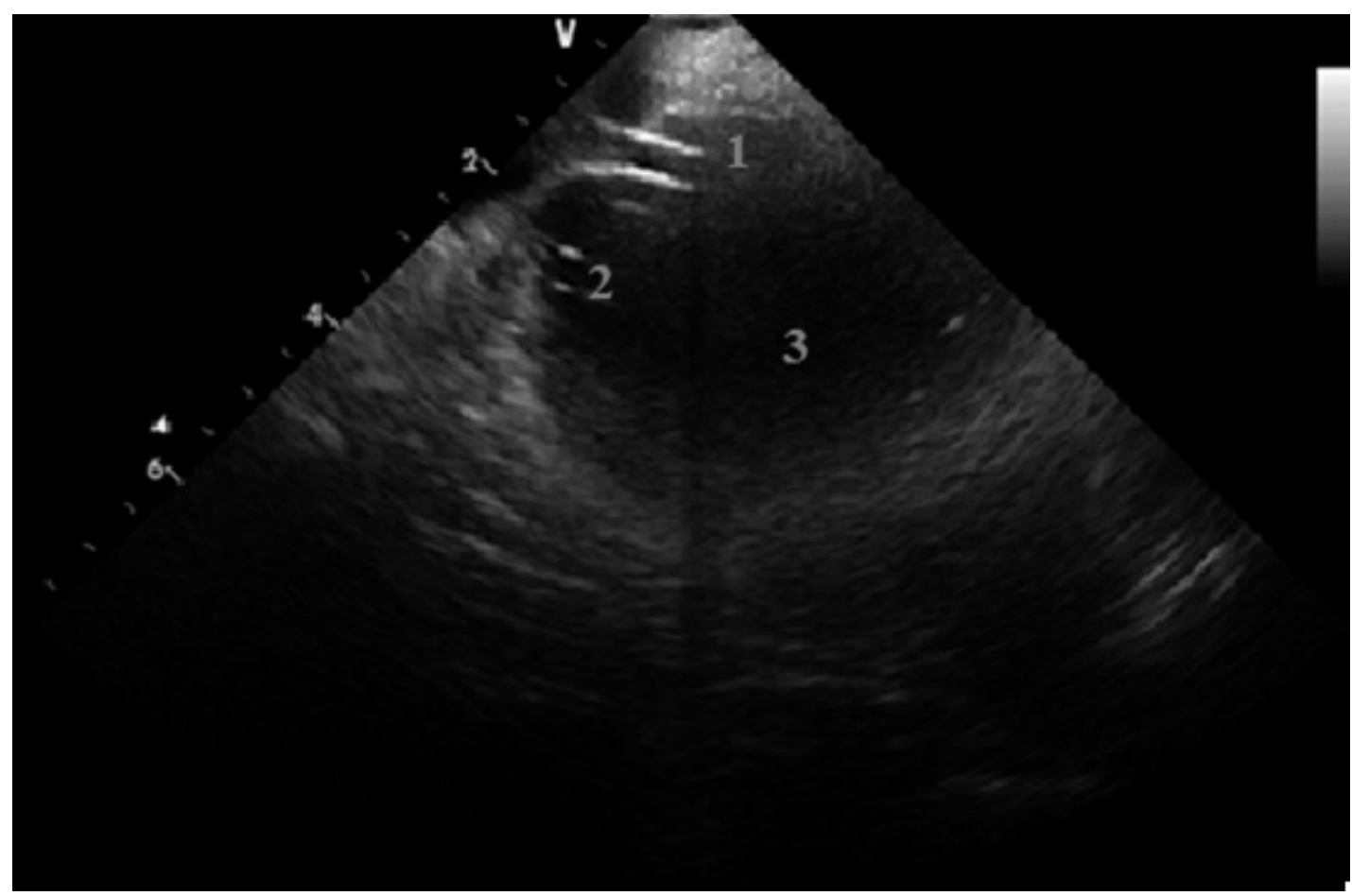

Рис. 5. позиционирование инструментов в ЛП

Примечание 1,2 - интродьсеры в ЛП, 3 полость ЛП. Note: 1,2 - introducers in left atrium, 3 - left atrium. 


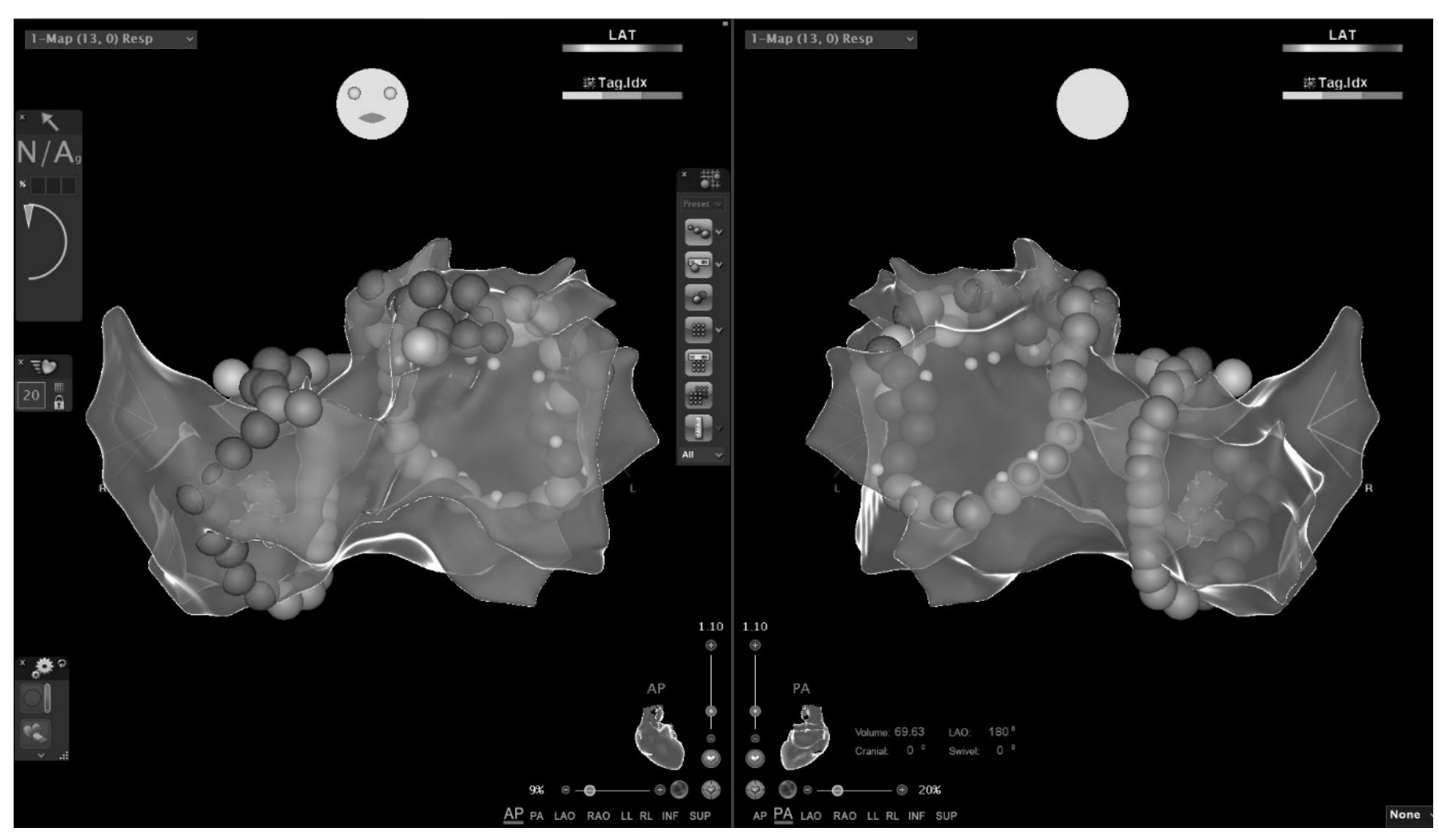

Рис. 6. Анатомическая карта изоляция устьев легочных вен

Примечание слева передняя проекция, справа задняя проекция. Note: anterior-posterior view in the left, posterior-anterior view in the right.

Средний возраст 63+3 года. Сопутствующие заболевания и краткая характеристика пациентов представлена в таблице № 1. Пациенты были включены в исследования после информированного письменного согласия. Исследование одобрено этическим комитетом РУдН. Антикоагулянтная терапия проводилась минимум за 4 недели до операции. При терапии варфарином, операция выполнялась при значении МНO 2,1-2,3. Операции проводились без отмены новых антикоагулянтов. Пациенты прекращали прием антиаритмических средств за 2-3 периода полувыведения перед абляцией. Перед операцией всем пациентам выполнялась трансторакальная и внутрисердечная эхокардиография (ЭХО-КГ) с целью исключения структурного заболевания сердца и образование тромба в левом предсердии и его ушке. По показаниям была проведена коронарография.

Ход операции: На первом этапе коронарный синус через яремный доступ. Трижды пунктировалась правая бедренная вена. После пункции бедренной вены проводилась системная гепаринизация (100 мг/кг).

Длинные проводники позиционировались в области верхней полой вены. Контроль положения проводников осуществлялся с помощью внутрисердечного
ЭХО-КГ (рис. 1). Для проведения внутрисердечного ЭхоКГ использовался аппарат GE Vivid-I, также датчик для внутрисердечного ЭХО-КГ ACUSON AcuNav ${ }^{\text {TM }}$ Ultrasound Catheter. Проведение интродьюсеров в верхнюю полою вену осуществлялось под контролем внутрисердечного ЭХО-КГ (рис. 2).

Пункция межпредсердной перегородки (МПП) проводилась под контролем внутрисердечного ЭХО-КГ (рис. 3). МПП пунктировалась максимально низко, кончик транссептальной иглы позиционировался на уровне «карины» между левыми легочными венами. После проведения пункции в полость левого предсердия (ЛП) вводился дилятор на расстояние примерно 2-3 мм. Транссептальная игла вынималась. Далее вводился длинный проводник и позиционировался в левую верхнюю легочную вену (рис. 4). По такой же схеме осуществлялась вторая пункция. Далее полость ЛП проводились рабочие инструменты (рис. 5).

Следующим этапом было построение анатомической карты в режиме быстрого построения. Использовалась система трехмерной навигации Carto $3^{\text {тм }}$ biosense webster. Использовался абляционный электрод с возможностью измерения силы прижатия - ThermoCool $^{\circledR}$ 


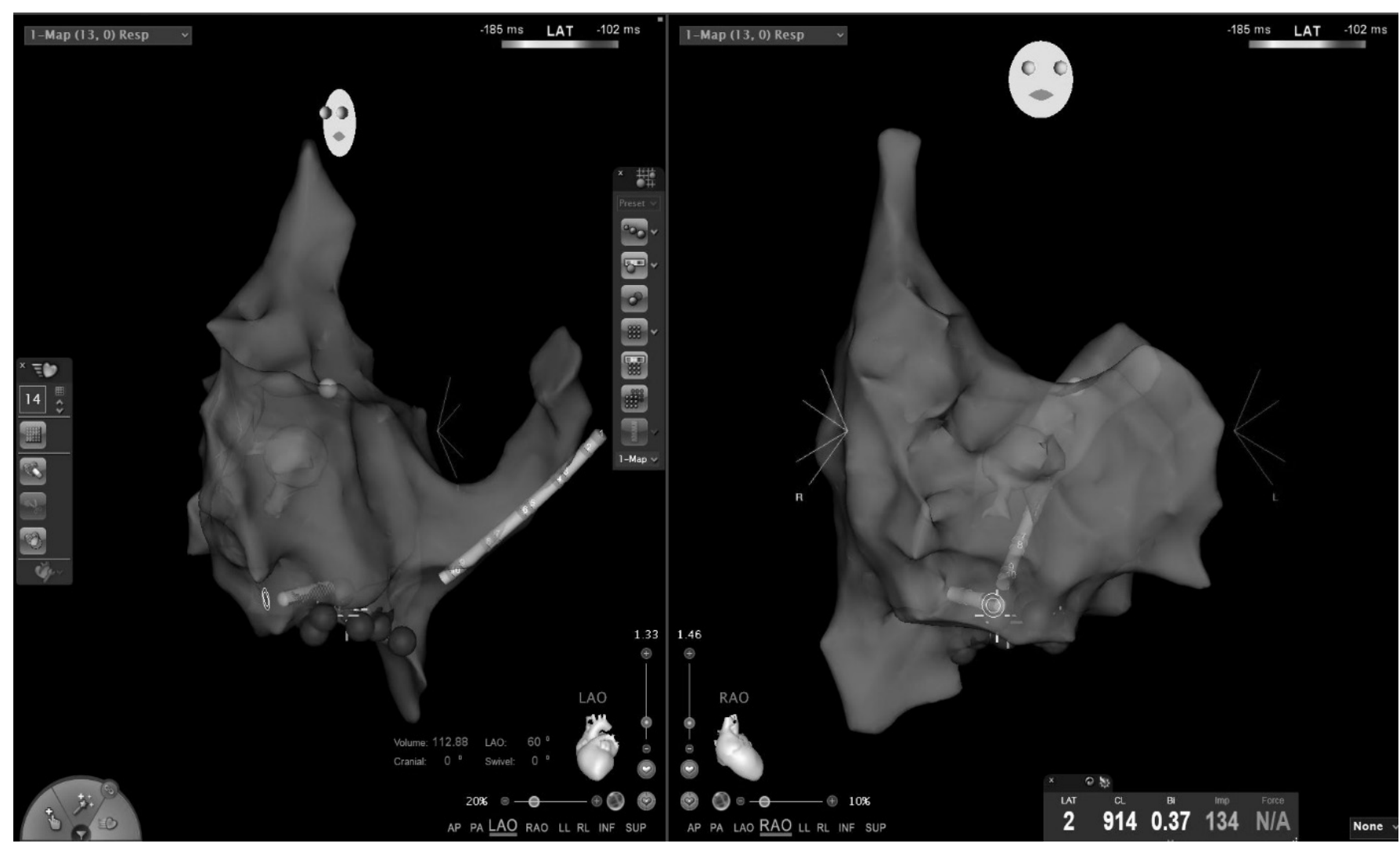

Рис. 7. Линейное воздействие по КТП

Примечание слева левая косая проекция, справа правая косая проекция. Note: left lateral obelic in the lest, right anterior obelic in the right.

SmartTouch ${ }^{\circledast}$ производство biosense webster. Далее проводилась антральная изоляция легочных вен. Использовался диагностический двадцатиполюсной электрод Lasso производство biosense webster, период ожидания после воздействия 20 минут. Изоляция легочных вен оценивалась по следующим параметрам: уменьшение амплитуды сигнала с диагностического электрода более чем в 5 раз, отсутствие проведение импульса (блок входа и блок выхода). После удаления инструментов из левого предсердия мы измеряли время правопредсердного проведения. Далее представлен рис. 6 после проведения изоляции легочных вен.

После проведения изоляции легочных вен диагностический электрод удалялся, абляционным электродом проводилось построение анатомической карты правого предсердия. Далее 10 полюсной электрод (WEBSTER ${ }^{\circledR}$ Decapolar Deflectable Catheter) позиционировался в коронарный синус. Следующим этапом проводилась абляция КТП. На рис 8. Показаны используемые проекции и видно линейное воздействие по КТП. Конечной точкой процедуры абляции было достижение полного двунаправленного блока в КТП. После проведения абляции КТП операция заканчивалась.
Период наблюдения составил 12 месяцев. Последующее наблюдение за пациентами проводилось через 24 ч после процедуры с ЭКГ-мониторингом. Антикоагулянтная терапия возобновлялась через 6 часов после операции. Все пациенты продолжали антикоагулянтное лечение не менее 3 месяцев. Пациентам с рецидивирующим ФП в течение 3 месяцев после абляции была проведена кардиоверсия, и по показаниям был назначен антиаритмический препарат. Все пациенты проходили клиническую оценку у одного и того же врача через 3, 6, 12, месяцев, включая ЭКГ по 12 отведениям и 2-дневные записи по Холтеру. Через 3 месяца наблюдения мы отменяли антиаритмические препараты, если у пациентов не было ФП и ТП на 2-дневных ХМ-ЭКГ. Пациентам проводилась повторная абляция, если после трех месяцев сохранялись симптомные пароксизмы ФП или ТП.

\section{Результаты}

Критерии изоляции легочных вен удалось достичь у всех пациентов. Абляция КТП была успешно проведена у всех пациентов. Среднее время операции составило $125 \pm 14$ минут. Осложнения представлены ге- 
матомой в области пункции бедренной вены у одного пациента (3.13\%).

Период отдаленного наблюдения составил 12 месяцев. Случаев типичного трепетания предсердий диагностировано не было. Эффективность процедуры по поводу ТП составила $100 \%$ в течение одного года. Эффективность процедуры по поводу фибрилляции предсердий в течение 12 месяцев составила 78.3\% (пароксизмы ФП и ТП отсутствовали у 25 пациентов). У 7 пациентов (22,35\%) по данным двухдневного ХМ-ЭКГ были зарегистрированы эпизоды фибрилляции предсердий спустя 4 месяца после операции. Этим пациентам была повторно проведена изоляция легочных вен (операция проводилась на 4 месяце после операции). Интраоперационно у этих пациентов был определен прорыв линии блока в области левых легочных вен, их передней стен- ки. Также этим пациентам мы проверяли двунаправленный блок в КТП.

\section{Обсужление}

Наша работа показывает, что проведении изоляции легочных вен одномоментно с абляцией КТП без использования рентгеноскопии безопасна и эффективна при краткосрочном наблюдении. Однако необходимо проведение проспективного рандомизированного исследования с целью сопоставления результатов операции, времени операции и возможных осложнений.

\section{Выво $\triangle \mathrm{b}$}

Проведение сочетанной операции, а именно абляции КТП с изоляцией легочных вен без использования рентгеноскопии возможно и безопасно.

\section{ЛИТЕРАТУРА}

1. Feasibility of zero or near zero fluoroscopy during catheter ablation procedures: Laurent M. Haegeli, Linda Stutz, Mohammed Mohsen, Thomas Wolber, Corinna Brunckhorst, Chol-Jun 0n, Firat Duru Cardiology Journal 2019; 26(3):226-232. doi: 10.5603

2. A New Technique for Zero Fluoroscopy Atrial Fibrillation Ablation Without the Use of Intracardiac Echocardiography: Silvia Guarguagli, Ilaria Cazzoi, Aleksander Kempny JACC Clinical Electrophysiology 4(12):1647-1648. doi: 10.1016

3. Fluoroless and contrast-free catheter ablation without a lead apron in routine clinical practice: J. Mauricio Sánchez, Margaret A. Yanics, Patricia Wilson, Amit Doshi, Thomas Kurian, Stephen Pieper. Journal of Interventional Cardiac Electrophysiology 08/2/2016

4. Zero Fluoroscopy Ablation: Recent Trends in Radiation Exposure in the EP Lab: Alaina R. Martinez, Chris Anderson, Mansour Razminia. Current Treatment Options in Pediatrics 11/2019 D0I: 10.1007/s40746-019-00170-7

5. Zero Fluoroscopy Ablation of Atrial Fibrillation: A Safety and Feasibility: Luigi Di Biase, Rodney Horton, Chintan Trivedi, Prasant Mohanty, Sanghamitra Mohanty, Rong Bai, Javier Sanchez, G. Joseph Gallinghouse, Jason Zagrodzky, Amin Al-Ahmad, Patrick Hranitzky, J. David Burkhardt, Andrea Natale. Circulation 27 Mar 2018 doi: $10.5603 /$ CJ.a22018.0029

6. Zero-Fluoroscopy Ablation at the Texas HeartInstitute: David Burkland, MD Tex Heart Inst J (2020) 47 (1): 53-56. doi: 10.14503/THIJ-19-7057

7. Near-zero x-ray in arrhythmia ablation using a 3-dimensional electroanatomic mapping system: A multicenter experience: Marzia Giaccardi, Attilio Del Rosso, Vincenzo Guarnaccia, Piercarlo Ballo, Giuseppe Mascia, Leandro Chiodi, Andrea Colella Heart Rhythm 2016 Jan;13(1):150-6. doi: 10.1016/j.hrthm.2015.09.003.

8. Safety profile of near-zero fluoroscopy atrial fibrillation ablation with non-fluoroscopic catheter visualization: experience from 1000 consecutive procedures Philipp Sommer: Livio Bertagnolli, Simon Kircher, Arash Arya, Andreas Bollmann, Sergio Richter, Sascha Rolf, Gerhard Hindricks Europace. 2018 Dec 1;20(12):1952-1958. doi: 10.1093/europace/eux378.

9. Zero-fluoroscopy approach for ablation of supraventricular tachycardia using the Ensite NavX system: a multicenter experience Guangzhi Chen, Yan Wang, Riccardo Proietti, Xunzhang Wang, Feifan Ouyang, Chang Sheng Ma, Rong Hui Yu, Chunxia Zhao, Kezhong Ma, Jie Qiu, Qigong Liu, Dao Wen Wang Chen et al. BMC Cardiovascular Disorders (2020) doi.org/10.1186/s12872-020-01344-0.

10. Occupational radiation exposure in the electrophysiology laboratory with a focus on personnel with reproductive potential and during pregnancy: A European Heart Rhythm Association (EHRA) consensus document endorsed by the Heart Rhythm Society (HRS) Andrea Sarkozy, Tom De Potter, Hein Heidbuchel, Sabine Ernst, Jedrzej Kosiuk, Eliseo Vano, Eugenio Picano, Elena Arbelo, Usha Tedrow EP Europace, Volume 19, Issue 12, December 2017, Pages 1909-1922. doi. org/10.1093/europace/eux252

11. Zero-fluoroscopy approach for ablation of supraventricular tachycardia using the Ensite NavX system: a multicenter experience Guangzhi Chen, Yan Wang, Riccardo Proietti, Xunzhang Wang, Feifan Ouyang, Chang Sheng Ma, Rong Hui Yu, Chunxia Zhao, Kezhong Ma, Jie Qiu, Qigong Liu, Dao Wen Wang. BMC Cardiovasc Disord. 2020; 20: 48 Published online 2020 Feb 3. doi: 10.1186/s12872-020-01344-0 